VOL. $31(1985), 317-318$.

\title{
A STUDY OF KNOT-GRAPHS
}

\author{
John Christopher TURner
}

This thesis is an account of studies made of knot projections, the tools used being graph-theoretic methods and extensions of them. The basic tool is the adjacency matrix. In fact several kinds of adjacency matrix are defined for knot diagrams which are either nonoriented or are given orientations which reflect certain topological properties of the knots concerned.

First the properties of knot-graph adjacency matrices are studied. In particular, a matrix equation is derived which is shown to be satisfied by the $\alpha$ - and $\beta$-adjacency matrices of all knot-graphs; other matrices appearing in the equation are studied.

Examination of types of walk that can be made on a knot-graph leads to a definition of walk-groups and groupoids on knot-graphs. Relations between the Cayley diagrams of walk-groups and certain other knots which are derived from the diagrams lead to interesting correspondences between knots, both finite and infinite.

An extensive study of the spectra of knot-graph adjacency matrices is presented. Methods for obtaining spectra are given, both for general cases and for certain classes of knots.

The concept of balanced orientations of knot-graphs is introduced; and numbers of rooted directed spanning trees of oriented knot-graphs are studied. It is shown that one type of tree number is a knot invariant, and

Received 12 December 1984. Thesis submitted to University of Waikato, March 1984. Degree approved November 1984. Supervisors: Professor A. Zulauf and $\mathrm{Dr} \mathrm{K}$. Broughan.

Copyright Clearance Centre, Inc. Serial-fee code: ooo4-9727/85 $\$ \mathrm{~A} 2.00+0.00$. 
evidence for the invariance of another one is given. A general inequality relating the two types of number is conjectured. The first tree number has several topological interpretations, and these are discussed. Tables of the numbers are given for knots of orders $n=3, \ldots, 10$; and methods for calculating them for various knot classes are developed.

A vertex deletion theorem relating tree numbers of three knot-graphs is proved. Families of twins which arise from vertex deletions in knotgraphs are defined and studied. It is shown that by repeatedly applying the deletion operation on a given alternating knot-graph, one is led to a certain distribution of 'twists', which is given the name 'twist spectrum'. Twist spectra have many interesting topological properties: they are topological invariants, and their moments are powerful knot discriminants; they are topological invariants, and their moments are powerful knot discriminants; they distinguish between a knot-graph and its mirror image and hence can be used to investigate amphicheirality.

The twist spectra for prime alternating l-links up to order $n=9$ and for 2-links up to order $n=8$ are tabulated. A general algorithm for computing a twist spectrum is given, and formulae are obtained for twist spectra of members of certain knot-classes.

Department of Mathematics, University of Waikato, Hamilton, New Zeal and. 